\section{SPRING BOOK REVIEWS}

All titles reviewed here are available from the BMJ Bookshop, PO Box 295, London WC1H 9TE. Prices include postage in the UK and for members of the British Forces Overseas, but overseas customers should add $15 \%$ to the value of the order for postage and packing. Payment can be made by cheque in sterling drawn on a UK bank, or by credit card (Mastercard, Visa, or American Express) stating card number, expiry date, and full name.

Genetics. J M Friedman, F J Dill, M R Hayden, B C McGillivray. (Pp 250; $£ 13.95$.) London: Williams \& Wilkins. 1992

I suspect this book must be read as one of the many titles in the National Medical Series for Independent Study. This is a list of texts produced to aid students prepare for predominantly United States Medical Board examinations. Genetics, we are told, belongs to the basic science rather than the clinical science series. You may perceive I began to have doubts about this publication even before I had left the preface behind.

What follows are 198 pages of note form factual information. Chapters move from gene structure and chromosomal organisation to population genetics to techniques of DNA diagnosis and finally to two chapters on clinical genetics. One has to say that the book is quite a remarkable achievement on the part of the four authors, all colleagues in the Medical Genetics Department at the University of Vancouver. However, this close working relationship has not prevented a number of irritating repetitions and, given the pressure for space, these should be weeded out in a future edition. The pages are crammed with power punching information; blink and the technique of PCR will be lost on the unsuspecting. Indeed it is for such a novice that my heart bleeds. I am bewildered to imagine either a medical student or recent undergraduate (whom the authors state to be the intended audience) leaving this volume both confident, or perhaps more importantly satisfied, in their endeavours to embrace modern clinical genetics.

On the positive side, the book is heaven sent to the student who prefers to hang on every word of his course tutor rather than take lecture notes. The content is comprehensive and I noted few errors. It would also be welcome to course tutors as a useful source of examination questions; the final section provides over 150 questions and answers relevant to the text. The book is up to date in a field moving faster than any other in medicine. However, its brevity withou clues to source information or original references must leave the more inquisitive reader less than inspired. The authors state they "hope to encourage all aspiring physicians to share in the excitement of thinking genetically". I must express reservations that this text will have limited value in contributing to this important objective.
Prader Willi Syndrome and Other Chromosome 15q Deletion Disorders. NATO ASI Series H Cell Biology Volume 61. Edited Suzanne B Cassidy. (Pp 265; DM 198.) Berlin: Springer-Verlag. 1992

The list of titles preceding this volume in the NATO Advanced Science Institution Series $\mathrm{H}$ (cell biology) contains expressions such as "signal transduction . . . cell to cell junctions translational apparatus" which seem surprising bedfellows for a volume on chromosome $15 \mathrm{q}$ deletion disorders. I guess the cell biological connection lies in the term 'imprinting', a concept which has recently acquired quasi-religious significance for the clinical geneticist. Fortunately, many papers given at the first international workshop (May 1991) on Prader-Willi syndrome (PWS) and related disorders and collected together in this volume make no mention of imprinting and instead provide an up to date survey of clinical, cytogenetic, and molecular studies currently in progress. Although clinical aspects of PWS are given extensive coverage, considerably less space is devoted to Angelman syndrome. I suppose this may reflect current research activities and thus indicate potential for further clinical studies of the latter important disorder. Several patients with proximal 15q deletions, but neither of the above clinical syndromes, are discussed in a single detailed contribution. Naturally PWS and Angelman syndrome are compared and contrasted in papers dealing with cytogenetic and molecular genetic findings. It is very helpful to have data from different research groups published back to back and it can be seen that no major discrepancies arise, although much remains unexplained. From my personal point of view, a conclusion worth emphasising is that careful clinical diagnosis remains crucial in PWS and Angelman syndrome. In summary, this volume will be useful in the clinic and in the laboratory for tackling diagnostic problems and for assessing the utility of molecular genetic investigations. However, its main value lies in graphically illustrating how little we actually know, so I believe anyone seeking an account of the cell biology of PWS and Angelman syndrome will be disappointed. Perhaps NATO, having been relieved of weightier responsibilities, could be persuaded to sponsor a fresh appraisal of these two syndromes in five or 10 years time. A report of the meeting and abstracts of contributions to it were published in the American fournal of Medical Genetics, volume 42, pp 220-69.

JOHN TOLMIE

The Code of Codes. Scientific and Social Issues in the Human Genome Project. Edited D J Kevles, Leroy Hood. (Pp 397; £23.95.) London: Harvard University Press. 1992.

Readers of the Journal will be well acquainted with the technology and medical implications of the Human Genome Project. But perhaps not everyone has considered all the social, ethical, and legal consequences, and no doubt fewer will be aware of the political infighting which the Project has engendered. All these matters are discussed at great length in this new book of some 14 chapters, divided into three parts: History and politics, Technology, and Ethical and legal considerations.

The Project officially began in 1991 and is estimated to be completed in possibly 10 to 15 years time. One contributor believes that within 10 years, with advances in instrumentation and technology, a single technician may be able to sequence $10^{6}$ base pairs a day! And currently the costs are put at around a dollar a base pair. The contributors are all enthusiastic that this concentration of effort and resources is well justified, but it would have been valuable to have had the views of those who are not so enthusiastic, of which there are, of course, many on both sides of the Atlantic.

Important ethical, legal, and social problems will be generated by the Project: matters of confidentiality, discrimination by employers, stigmatisation by society, sharing of results, and patenting of sequences, etc. To address these and similar concerns NIH has allocated $3 \%$ of its genome budget. Some consider this as evidence of a genuine concern with these matters. Others, I suspect, will realise that to really embark on any meaningful programme will involve considerably more expenditure as well as an ongoing commitment.

Many of the contributors are internationally known experts and write with authority. They include, for example, Kevles (whose In the Name of Eugenics (Penguin) has become a classic), Judson, Gilbert, Caskey, and Nancy Wexler. James Watson's 'Personal View' is particularly revealing when one realises that Bernadine Healy, who is now head of NIH, is replacing him as head of the Project.

As with many multi-authored texts, there is often overlap and repetition and there is occasionally an element of chauvinism. But, having said this, there is much to interest and provoke thought in this new text.

\section{ALAN EMERY}

The Atlas of Mouse Development. Matthew H Kaufman. (Pp 512; $£ 80.00$.) London: Academic Press. 1992.

This splendid volume is rather grandly called The Atlas of Mouse Development. Given the author's approach it is difficult to see anyone else going through the exercise again, so that the title is probably justified. The aim of the book is to "provide an histological account of the development of the mouse spanning the period from fertilization to term". The method used is to assemble a comprehensive collection of meticulously prepared and labelled histological sections at different gestations. The book is intended as a 'workshop manual' rather than a text book of embryology. As the author points out, it is difficult to reconstruct a three dimensional image in the mind from a series of sections. Although he recommends the exercise as ultimately rewarding, only those directly involved in studying mouse embryos will want to invest in the considerable effort needed to attain this level of understanding.

The plates would have been better in colour, but again this would have made the price of the book prohibitive. Perhaps in the future some form of electronic publication, for example on CD-ROM, could be explored There is also a collection of scanning EM photographs illustrating the development of different organ systems (for example, the palate, the eye, the limbs). These would be 
very useful as a source of illustrations for lectures to students on normal embryology in relation to malformations.

Altogether a magnificent achievement, and a volume that will be widely used as in situ hybridisation with molecular probes for 'developmental genes' becomes a standard procedure. This is not a book for the clinical genetics department but I imagine it should be the bible for developmental biologists.

\section{R M WINTER}

Neurofibromatosis: Phenotype, Natural History and Pathogenesis. 2nd edition. V M Riccardi. (Pp 498; \$68.50.) Baltimore: Johns Hopkins University Press. 1992.

When the first edition of Dr Riccardi's monograph on neurofibromatosis was published in 1986, work on the molecular genetics of the neurofibromatoses was only just beginning. The NF1 gene has now been cloned and scientists are beginning to elucidate the pathogenetic mechanisms of the disease; the NF2 gene has been localised to such a small part of chromosome 22 that its cloning must be considered to be imminent. The last six years has also seen a continuing increase in the number of clinical papers about the different types of neurofibromatosis published each year. Much of the credit for stimulating this interest in the disease must go to Dr Riccardi, both through his extensive publications and personal encouragement to young investigators when they first enter the field.

With this recent rapid increase in knowledge an updated second edition of the monograph is timely. The revised layout of the book reflects the increased knowledge, in that in the 1986 edition there was just one chapter on pathogenesis, where there are now four separate chapters covering molecular biology, cell biology, clinical biology, and animal models. In the clinical sections of the book, recent publications have been widely reviewed and the appropriate sections updated or expanded. The bibliography at the end of the book is extensive.

My only criticism is that on some of the sections on clinical management, a thorough discussion of the pros and cons of various management options is not presented. One of the examples of this is whether patients with neurofibromatosis type 1 should have routine cranial imaging to detect asymptomatic optic gliomas. Dr Riccardi is in no doubt that this is indicated and comments ". . . it seems clear that until physicians who are immediately responsible for the health care of these children adopt a publicly stated, aggressive 'go get 'em' attitude, dozens if not hundreds of children with NF1 will unnecessarily lose at least a portion of their sight each year". Many other clinicians in the field would not agree that symptomatic optic gliomas are frequent enough to warrant routine cranial imaging in children with NF1.

At the time of the first edition in 1986, there were no other recent books about neurofibromatosis. In 1990, a multi-author handbook on neurofibromatosis was published for both patients with the disease and health care professionals (Neurofibromatosis. $A$ handbook for patients, families, and health care professionals. Eds Rubenstein AE, Korf BR. New York: Thieme Medical Publishers,
1990). The two books complement one another as they are aimed at different audiences. If I had to choose, as a clinical geneticist, which to purchase at present, it would be the Riccardi monograph because of its more in depth coverage of research aspects and the extensive bibliography. However, for health professionals caring for NF families and their patients, the Rubenstein and Korf book is a good introductory text. For anyone embarking on scientific and clinical research of neurofibromatosis, the Riccardi book, particularly in view of its exhaustive bibliography, is a must.

SUSAN M HUSON

Genetic Disorders and the Fetus. Diagnosis, Prevention and Treatment. 3rd edition. Editor A Milunsky. ( $\mathrm{Pp}$ 336; $\$ 111.50$.) Baltimore: Johns Hopkins University Press. 1992.

Six years have elapsed since the second edition of this standard textbook in the field of prenatal diagnosis was published. These years have seen a particularly rapid growth in the number of applications of prenatal molecular genetic diagnosis and in the development of maternal biochemical screening for fetal aneuploidies in the first and second trimesters of pregnancy. A new edition was thus essential and, reflecting the multidisciplinary nature of the subject, 48 distinguished contributors were recruited from a diversity of clinical and scientific disciplines.

The new edition succeeds in its goal of providing extensive critical discussion of the state of the art in prenatal diagnosis and the editor and publishers are to be congratulated on bringing a project of this scale so rapidly into print. My two main criticisms relate to imbalanced coverage of disorders and to areas of omission. I suspect the former is historical but the result is comprehensive coverage of chromosomal and biochemical disorders with relative neglect of other genetic disorders. Thus, for example, cystinosis has over one page which details the clinical, genetic, and biochemical features whereas Duchenne muscular dystrophy and Huntington's disease are covered much more briefly and the emphasis mainly relates to molecular diagnosis. Surprising omissions are the psychosocial sequelae of termination of pregnancy for fetal abnormality and the aspects of clinical management which can influence these sequelae; inherited serious skin disorders and the role of fetal skin biopsies; and no mention in the index of a variety of rarer disorders for which prenatal diagnosis is available. This last omission could be rectified by including an appendix of conditions for which prenatal diagnosis has been reported and so avoid the potential user error that lack of inclusion reflects lack of availability of a prenatal test.

The pace of progress in the field of prenatal diagnosis and screening is relentless with over 500 publications a year. This textbook has a track record for successful evolution to meet this challenge and this should ensure its continued inclusion in the libraries of those who are actively involved in prenatal diagnosis research and practice.

J M CONNOR
Micromanipulation of Human Gametes and Embryos. J Cohen, $\mathrm{H}$ E Malter, B Talansky, J Grifo. New York: Raven Press. 1992.

This book is a mixture of subject review and hands on guide. The first five chapters are excellent reviews within the field of micromanipulation in developmental biology and the later chapters are presented more in the mode of a techniques manual. There is some confusion in the organisation of these sections especially in the second half of the book. Despite this, both the review and techniques chapters in themselves are presented in clear and consistent fashion and with excellent use of illustrative diagrams and photographs throughout. The coverage of past work on the development of the ideas and procedures is comprehensive. This makes interesting reading and it is pleasing to see the original work acknowledged.

Chapter 1 gives an interesting historical perspective of micromanipulation over 150 years from hair loops (still used today) to the most sophisticated instrumentation now available. I would like to comment at this point that there might be a tendency today to rely too much on sophisticated (and expensive) equipment and thus to bypass our own inherent skills which are developed by working always with the minimum assistance from instrumentation and the lowest magnification possible. It is truly amazing the skills I have seen developed by persons who, with perseverance, practice, and acclimatisation to the microenvironment, regularly produce hand crafted pipettes with 5 micron apertures and who accomplish reliable single cell work using only a simple dissecting microscope. Conversely, I have visited laboratories where it takes hours or days to set up the micromanipulation apparatus to perform a task that could be done in minutes with a dissecting microscope. Micromanipulation then can be saved for the most delicate tasks which cannot be done by hand and chapters 2 and 3 cover its application to these tasks in the fields of developmental embryology and animal breeding. Chapter 4 describes gametogenesis and fertilisation and chapter 5 homes in on the different approaches to microsurgical fertilisation in mammals. Microsurgical fertilisation may be a treatment for some forms of infertility which have not been amenable or suitable for regular IVF procedures and this is the main research interest of the authors. The following chapters present the practical approaches to microsurgical fertilisation (chapter 6) and their evaluation (chapter 7), consequences of zona pellucida micromanipulation (chapter 8), and a comprehensive coverage of equipment, tools, and techniques (chapter 10). This covers nearly all of the micromanipulation procedures which are currently applied to human gametes and embryos except for oocyte and embryo biopsy, so important in the rapidly developing field of preimplantation diagnosis of genetic disease. Unfortunately, embryo biopsy is not adequately covered in the rather poorly written chapter 9 in which detailed procedures are given only for gel electrophoresis and fluorescent in situ hybridisation.

Other micromanipulation procedures, such as disaggregation and cloning, nuclear transplant, gene injection and transgenesis, aggregation and chimaerism and embryonic stem cell technology are not covered technically in this book. Such procedures are rightly limited to mouse models and animal 